\title{
Prolyl hydroxylase domain inhibitors: a new era in the management of renal anemia
}

\author{
Yu Kurata, Tetsuhiro Tanaka, Masaomi Nangaku \\ Division of Nephrology and Endocrinology, The University of Tokyo Graduate School of Medicine, Tokyo, Japan \\ Correspondence to: Masaomi Nangaku. Division of Nephrology and Endocrinology, The University of Tokyo Graduate School of Medicine, 7-3-1 \\ Hongo, Bunkyo-ku, Tokyo 113-8655, Japan. Email: mnangaku-tky@umin.ac.jp. \\ Provenance: This is an invited article commissioned by the Section Editor Dr. Linpei Jia (Department of Nephrology, Xuanwu Hospital of Capital \\ Medical University, Beijing, China). \\ Comment on: Chen N, Hao C, Peng X, et al. Roxadustat for Anemia in Patients with Kidney Disease Not Receiving Dialysis. N Engl J Med \\ 2019;381:1001-10.
}

Submitted Sep 08, 2019. Accepted for publication Sep 20, 2019.

doi: 10.21037/atm.2019.09.118

View this article at: http://dx.doi.org/10.21037/atm.2019.09.118

Prolyl hydroxylase domain (PHD) inhibitors are orally bioavailable small-molecule compounds that activate hypoxia inducible factor (HIF). HIF is a master transcription factor for regulating the cellular adaptive response to hypoxia. Erythropoietin (EPO), a hormone essential for red blood cell production, is one of the target genes of HIF. PHD inhibitors have been developed for the treatment of anemia with chronic kidney disease (CKD). There are several advantages of PHD inhibitors over conventional erythropoiesis stimulating agents (ESAs): oral administration, lower cost, physiological levels of endogenous EPO production, and improved iron utilization efficiency. Several PHD inhibitors (roxadustat, vadadustat, daprodustat, molidustat, enardustat, and desidustat) are currently under phase III studies. Among them, roxadustat has gained an advantage in the development race. The first phase III study of roxadustat carried out in China that involved CKD patients not on dialysis with renal anemia was reported by Chen et al. in July 2019 (1). Following the results of this trial, China has become the first country to approve roxadustat for the treatment of anemia in CKD for both on dialysis and not on dialysis patients.

\section{Renal anemia and ESAs}

The number of patients with CKD has been rapidly increasing throughout the world. The burden of CKD has drawn global attention. Renal anemia is a major complication for patients with CKD and is associated with an impaired quality of life as well as cardiovascular diseases (CVDs). The kidney is the primary source of EPO production, and EPO deficiency is the main cause of renal anemia. Since the 1980s, ESAs have drastically changed the management of renal anemia. ESAs have reduced the frequency of blood transfusion, resulting in decreased transfusion-related complications. Observational studies have reported improved quality of life and exercise capacity as beneficial effects of anemia treatment by ESAs. Although ESAs have been the mainstay of anemia treatment in CKD patients, there are drawbacks regarding the use of ESAs: painful injections in patients not on hemodialysis, hypertension, thromboembolic events, and increased CVDs. Another major concern in renal anemia treatment is ESA hyporesponsiveness, which is an insufficient response in hemoglobin levels despite administration of large amounts of ESAs. A variety of conditions such as iron deficiency, inflammation, and uremia may contribute to ESA hyporesponsiveness.

\section{HIF and PHD inhibitors}

HIF induces an array of target genes related to erythropoiesis, angiogenesis, and energy metabolism in response to hypoxic stress. HIF consists of two subunits: oxygen sensitive HIF- $\alpha$ and constitutively expressed HIF- $\beta$. Three isoforms of HIF- $\alpha$ have been identified: HIF- $1 \alpha$, HIF- $2 \alpha$, and HIF- $3 \alpha$. HIF- $\alpha$ is negatively regulated by PHDs depending on oxygen concentration. PHDs are 
members of the 2-oxoglutarate dependent dioxygenase family. PHDs require cofactors including an oxygen molecule, $\alpha$-ketoglutarate, iron, and ascorbate. PHDs have three isoforms (PHD1, PHD2, and PHD3) in which PHD2 regulates HIF- $\alpha$ expression. Under normoxic conditions, PHDs hydroxylate two proline residues of HIF- $\alpha$. Hydroxylated HIF- $\alpha$ is recognized by von-Hippel Lindau (VHL) protein, a component of the ubiquitin E3 ligase complex, thereby HIF- $\alpha$ is ubiquitinated, followed by proteasomal degradation. Under hypoxic conditions, PHDs fail to hydroxylate HIF- $\alpha$ proline residues. HIF- $\alpha$ translocates into the nucleus, where it forms a heterodimer with HIF- $\beta$ and upregulates various target genes with hypoxia response elements (HREs) in the promoter regions.

PHD inhibitors ameliorate anemia via increased EPO production and improved iron utilization efficiency with physiological levels of endogenous EPO, whereas exogenous ESAs increase the hemoglobin levels with supraphysiological concentrations of circulating EPO, which may lead to increased adverse events. As CKD progresses, EPO-producing cells in the kidney interstitium are transformed into myofibroblasts and lose the ability to secrete EPO. HIF activation allows these cells to regain EPO-producing capacity and, to a lesser extent, stimulates hepatic EPO production. Therefore, PHD inhibitors can effectively increase serum EPO levels in advanced CKD patients. Iron homeostasis is closely related to erythropoiesis, and HIF activation is implicated in iron metabolism. Dietary iron is reduced by duodenal cytochrome B (DCYTB) and is absorbed into intestinal epithelial cells via divalent metal transporter 1 (DMT1). In iron deficiency, stored iron in cells such as hepatocytes and macrophages is exported to the circulation via ferroportin (FPN), an iron transport membrane protein. FPN is regulated by hepcidin, a hormone produced by the liver. Circulating hepcidin binds to FPN on the cell surface. Hepcidin binding triggers endocytosis and lysosomal degradation of FPN, resulting in an impaired iron supply. During inflammation, hepcidin is induced by interleukin-6 (IL-6), an inflammatory cytokine. In patients with CKD, hepcidin levels are elevated because of decreased hepcidin excretion and chronic inflammation, which leads to impaired iron utilization and ESA hyporesponsiveness. The above key enzymes involved in iron homeostasis such as FPN, DCYTB, and DMT1 are upregulated by HIF- $2 \alpha$. Furthermore, HIF activation suppresses hepcidin production indirectly, possibly via erythroferrone which is a hormone secreted from erythroblasts in response to
EPO. The phase II studies of roxadustat have demonstrated a dose-dependent hemoglobin increase with physiological EPO levels in the blood. Roxadustat administration also decreased hepcidin and total cholesterol levels. Furthermore, roxadustat treatment increased hemoglobin levels independent of baseline C-reactive protein (CRP) levels, suggesting that roxadustat may be effective in patients with inflammation-induced ESA hyporesponsiveness.

\section{The results of the phase III study of roxadustat}

Roxadustat is the first-in-class PHD inhibitor that completed phase III studies. Chen at el. reported the results of the first phase III study of roxadustat in CKD patients not on dialysis with renal anemia in The New England Fournal of Medicine (NEFM) (1). The study consisted of two phases: an initial 8-week, double-blind, placebocontrolled phase and an 18-week open-label phase during which all patients received roxadustat. One hundred and fifty-four patients with stage G3-5 CKD not on dialysis were included: 102 in the roxadustat group and 52 in the placebo group. The results of the primary outcome, the mean change in the hemoglobin levels from baseline to weeks $7-9$, were as follows: an increase of $1.9 \pm 1.2 \mathrm{~g} / \mathrm{dL}$ in the roxadustat group and a decrease of $0.4 \pm 0.8 \mathrm{~g} / \mathrm{dL}$ in the placebo group. The rate of a hemoglobin response (an increase of $\geq 1 \mathrm{~g} / \mathrm{dL}$ ) in the roxadustat group was $84 \%$. In terms of iron metabolism, roxadustat decreased serum hepcidin levels. The decline in the hepcidin level from baseline was $56.14 \pm 63.40 \mathrm{ng} / \mathrm{mL}$ in the roxadustat group and $15.10 \pm 48.06 \mathrm{ng} / \mathrm{mL}$ in the placebo group. Roxadustat also had effects on lipid metabolism. The decrease in total cholesterol level was $40.6 \mathrm{mg} / \mathrm{dL}$ and the decrease in lowdensity lipoprotein (LDL) cholesterol levels was $25.3 \mathrm{mg} / \mathrm{dL}$ in the roxadustat group and 7.7 and $5.8 \mathrm{mg} / \mathrm{dL}$ in the placebo group, respectively. This finding is consistent with animal experiments that showed lipid-lowering effects of PHD inhibitors (2). During the open-label phase, the roxadustat group maintained stable hemoglobin levels, while the placebo group had an increase in hemoglobin levels, comparable to the roxadustat group. Taken together, the roxadustat treatment increased hemoglobin levels with decreased serum cholesterol and hepcidin levels compared with the placebo, which are consistent with the results of earlier phase II trials.

During the initial randomized phase, $68 \%$ in the roxadustat group and $75 \%$ in the placebo group had at least one adverse event. Compared with the placebo group, 
hyperkalemia $(>5.5 \mathrm{mmol} / \mathrm{L})$ and metabolic acidosis were reported more frequently in the roxadustat group: $16 \%$ vs. $8 \%$ and $12 \%$ vs. $2 \%$, respectively. In a phase III trial of roxadustat published in NEJM at the same time, in which 305 dialysis patients were involved, hyperkalemia was similarly reported frequently in the roxadustat group (3). In some phase II trials, hyperkalemia was reported more frequently as an adverse event in the PHD inhibitor group compared with the control group (4-6). Given that metabolic acidosis was also reported frequently in the roxadustat group, potassium shift from the intracellular space to the extracellular space caused by metabolic acidosis may have contributed to the development of hyperkalemia in the roxadustat group. There are several mechanisms that connect HIF activation to acid-base homeostasis. In hypoxia, mitochondrial oxidative phosphorylation is inhibited, and HIF activates target genes involved in glucose uptake and glycolysis, which leads to lactate accumulation via anaerobic glycolysis. In hypoxic tumor cells, carbonic anhydrase IX, one of the HIF target genes, contributes to extracellular acidification via increased conversion of $\mathrm{CO}_{2}$ to bicarbonate and proton, the latter of which is excreted outside of the cell (7). Conversely, HIF can act as a countermeasure against systemic acidosis. An experiment using liverspecific PHD2 knockout mice demonstrated that HIF activation in the liver enhanced liver gluconeogenesis from circulating lactate and protected against lactic acidosis (8). The effects of HIF activation on acid-base status are complicated. Further investigation is needed to determine the underlying mechanism of hyperkalemia and metabolic acidosis. In the phase III trial of roxadustat involving dialysis patients, noncardiac chest discomfort was reported frequently in the roxadustat group (3). Gastrointestinal symptoms including nausea and diarrhea were the most commonly reported adverse events in prior phase II trials, which may influence patients' adherence to treatment.

\section{Pleiotropic effects of HIF}

Vascular endothelial growth factor (VEGF) is a potent inducer of angiogenesis and contributes to tumor microenvironment. Although there have been no phase II trials reporting that PHD inhibitors significantly increase plasma VEGF level and PHD inhibitors are likely to induce erythropoiesis without an increase in systemic VEGF levels (9), there remain concerns about the potential risk of tumor progression and worsening retinal angiogenic conditions such as diabetic retinopathy and macular degeneration. Pulmonary hypertension is another concern. Patients with CKD are high-risk populations for pulmonary hypertension, and HIF- $2 \alpha$ has been implicated in the development of pulmonary hypertension.

In contrast, HIF stabilizers have potential as renoprotective agents by optimizing cellular adaptive responses to hypoxia. In the 5/6 nephrectomy model, a widely used CKD model, HIF activation exhibited renoprotective effects (10-12). However, in some experiments using conditional transgenic mice, HIF activation was associated with renal fibrosis progression. Proximal tubular-specific HIF-1 $\alpha$ knockout attenuated tubulointerstitial fibrosis following unilateral ureteral obstruction (UUO), a representative kidney fibrosis model (13). Proximal tubular-specific VHL knockout mice subjected to $5 / 6$ nephrectomy showed increased fibrosis after long-term observation (14). The effects of HIF activation on CKD progression may be cell typeand tissue context-dependent. Furthermore, in contrast to pharmacological intervention, the extent of gene activation in genetically engineered animals is supraphysiological, which may contribute to the discrepancy between these studies. In May 2019, FibroGen announced that in the pooled analysis of three roxadustat global phase III trials for nondialysis patients with baseline eGFR $\geq 15 \mathrm{~mL} /\left(\mathrm{min} \cdot 1.73 \mathrm{~m}^{2}\right)$, eGFR decline at 12 months in the roxadustat group was slower than that in the placebo group. The difference in eGFR decline between the groups was $1.62 \mathrm{~mL} /\left(\min \cdot 1.73 \mathrm{~m}^{2}\right)$. While this renoprotective effect of roxadustat may be attributed to increased hemoglobin levels, the influence of anemia treatment on CKD progression has been a matter of debate. A recent meta-analysis showed no beneficial effects of ESAs for the treatment of anemia on renal disease progression (15). Diseased kidneys suffer from a decrease in oxygen tension of resident kidney cells (16-19). Chronic hypoxia of the kidney is a final common pathway to end stage kidney disease (20-22) PHD inhibitors may prevent CKD progression by protecting the kidney against hypoxia directly.

\section{Conclusions}

PHD inhibitors are promising novel anemia treatment agents with advantages including oral administration, optimized iron metabolism, and physiological EPO secretion. For example, CKD patients with ESA hyporesponsiveness due to chronic inflammation and increased hepcidin levels may be good candidates for PHD inhibitors. The current guideline recommends 
that the normalization of hemoglobin level by ESAs be avoided. Large randomized controlled trials (RCTs) such as the Normal Hematocrit Cardiac Trial (NHCT) (23), Correction of Hemoglobin and Outcomes in Renal Insufficiency (CHOIR) (24), and Trial to Reduce Cardiovascular Events with Aranesp Therapy (TREAT) (25) demonstrated that targeting high hemoglobin level with ESA was associated with high CVD event rate. The secondary analyses of these trials suggested that high ESA doses required to treat ESA hyporesponsive patients rather than high target hemoglobin itself contributed to increased CVD risks. Considering that PHD inhibitors correct anemia with physiological levels of endogenous EPO, hemoglobin normalization by PHD inhibitors may improve clinical outcomes without increased CVD risks. Although no serious adverse events related to PHD inhibitors have been observed in prior clinical trials, the aforementioned safety concerns have not been sufficiently investigated due to a lack of long-term phase III data. Several global largescale phase III trials (NCT02052310, NCT02174627, and NCT02174731) are ongoing. The results of these trials and long-term post-marketing surveillance are essential to draw conclusions about safety issues.

\section{Acknowledgments}

Funding: The study was supported by the Grant-in-Aid for Scientific Research (B, C) from Japan Society for the Promotion of Science 18H02824 (M Nangaku) and 17K09688 (T Tanaka).

\section{Footnote}

Conflicts of Interest: M Nangaku has received honoraria, advisory fees, or research funding from Kyowa-Kirin, Akebia, Astellas, Chugai, GSK, JT, Taisho-Toyama, Torii, Mitsubishi-Tanabe, Daiichi-Sankyo, Bayer, and Boehringer Ingelheim. T Tanaka has received research grant from JT. The other author has no conflicts of interest to declare.

Ethical Statement: The authors are accountable for all aspects of the work in ensuring that questions related to the accuracy or integrity of any part of the work are appropriately investigated and resolved.

\section{References}

1. Chen N, Hao C, Peng X, et al. Roxadustat for Anemia in
Patients with Kidney Disease Not Receiving Dialysis. N Engl J Med 2019;381:1001-10.

2. Saito H, Tanaka T, Sugahara $M$, et al. Inhibition of prolyl hydroxylase domain (PHD) by JTZ-951 reduces obesity-related diseases in the liver, white adipose tissue, and kidney in mice with a high-fat diet. Lab Invest 2019;99:1217-32.

3. Chen N, Hao C, Liu B-C, et al. Roxadustat Treatment for Anemia in Patients Undergoing Long-Term Dialysis. N Engl J Med 2019;381:1011-22.

4. Besarab A, Provenzano R, Hertel J, et al. Randomized placebo-controlled dose-ranging and pharmacodynamics study of roxadustat (FG-4592) to treat anemia in nondialysis-dependent chronic kidney disease (NDDCKD) patients. Nephrol Dial Transplant 2015;30:1665-73.

5. Meadowcroft AM, Cizman B, Holdstock L, et al. Daprodustat for anemia: a 24-week, open-label, randomized controlled trial in participants on hemodialysis. Clin Kidney J 2019;12:139-48.

6. Pergola PE, Spinowitz BS, Hartman CS, et al. Vadadustat, a novel oral HIF stabilizer, provides effective anemia treatment in nondialysis-dependent chronic kidney disease. Kidney Int 2016;90:1115-22.

7. Svastová E, Hulíková A, Rafajová M, et al. Hypoxia activates the capacity of tumor-associated carbonic anhydrase IX to acidify extracellular $\mathrm{pH}$. FEBS Lett 2004;577:439-45.

8. Suhara T, Hishiki T, Kasahara M, et al. Inhibition of the oxygen sensor PHD2 in the liver improves survival in lactic acidosis by activating the Cori cycle. Proc Natl Acad Sci U S A 2015;112:11642-7.

9. Fukui K, Shinozaki Y, Kobayashi H, et al. JTZ951 (enarodustat), a hypoxia-inducibe factor prolyl hydroxylase inhibitor, stabilizes HIF- $\alpha$ protein and induces erythropoiesis without effects on the function of vascular endothelial growth factor. Eur J Pharmacol 2019;859:172532.

10. Tanaka T, Kojima I, Ohse T, et al. Cobalt promotes angiogenesis via hypoxia-inducible factor and protects tubulointerstitium in the remnant kidney model. Lab Invest 2005;85:1292-307.

11. Song YR, You SJ, Lee YM, et al. Activation of hypoxiainducible factor attenuates renal injury in rat remnant kidney. Nephrol Dial Transplant 2010;25:77-85.

12. Yu X, Fang Y, Liu H, et al. The balance of beneficial and deleterious effects of hypoxia-inducible factor activation by prolyl hydroxylase inhibitor in rat remnant kidney depends on the timing of administration. Nephrol Dial Transplant 
2012;27:3110-9.

13. Higgins DF, Kimura K, Bernhardt WM, et al. Hypoxia promotes fibrogenesis in vivo via HIF-1 stimulation of epithelial-to-mesenchymal transition. J Clin Invest 2007;117:3810-20.

14. Kimura K, Iwano M, Higgins DF, et al. Stable expression of HIF-1alpha in tubular epithelial cells promotes interstitial fibrosis. Am J Physiol Renal Physiol 2008;295:F1023-9.

15. Elliott S, Tomita D, Endre Z. Erythropoiesis stimulating agents and reno-protection: a meta-analysis. BMC Nephrol 2017;18:14.

16. Tanaka T, Miyata T, Inagi R, et al. Hypoxia in renal disease with proteinuria and/or glomerular hypertension. Am J Pathol 2004;165:1979-92.

17. Manotham K, Tanaka T, Matsumoto M, et al. Evidence of tubular hypoxia in the early phase in the remnant kidney model. J Am Soc Nephrol 2004;15:1277-88.

18. Tanaka T, Kato H, Kojima I, et al. Hypoxia and expression of hypoxia-inducible factor in the aging kidney. J Gerontol A Biol Sci Med Sci 2006;61:795-805.

19. Hirakawa Y, Mizukami K, Yoshihara T, et al. Intravital

Cite this article as: Kurata Y, Tanaka T, Nangaku M. Prolyl hydroxylase domain inhibitors: a new era in the management of renal anemia. Ann Transl Med 2019;7(Suppl 8):S334. doi: 10.21037/atm.2019.09.118 phosphorescence lifetime imaging of the renal cortex accurately measures renal hypoxia. Kidney Int 2018;93:1483-9.

20. Nangaku M. Chronic hypoxia and tubulointerstitial injury: a final common pathway to end-stage renal failure. J Am Soc Nephrol 2006;17:17-25.

21. Mimura I, Nangaku M. The suffocating kidney: Tubulointerstitial hypoxia in end-stage renal disease. Nat Rev Nephrol 2010;6:667-78.

22. Tanaka T, Nangaku M. Angiogenesis and hypoxia in the kidney. Nat Rev Nephrol 2013;9:211-22.

23. Besarab A, Bolton WK, Browne JK, et al. The effects of normal as compared with low hematocrit values in patients with cardiac disease who are receiving hemodialysis and epoetin. N Engl J Med 1998;339:584-90.

24. Singh AK, Szczech L, Tang KL, et al. Correction of Anemia with Epoetin Alfa in Chronic Kidney Disease. N Engl J Med 2006;355:2085-98.

25. Pfeffer MA, Burdmann EA, Chen C-Y, et al. A trial of darbepoetin alfa in type 2 diabetes and chronic kidney disease. N Engl J Med 2009;361:2019-32. 